\title{
INTERFACE MOTION IN DISORDERED FERROMAGNETS
}

\author{
L. ROTERS*, S. LÜBECK AND K. D. USADEL \\ Theoretische Tieftemperaturphysik, \\ Gerhard-Mercator-Universität Duisburg, 47048 Duisburg, Germany
}

February 28, 2019

\begin{abstract}
We consider numerically the depinning transition in the randomfield Ising model. Our analysis reveals that the three and four dimensional model displays a simple scaling behavior whereas the five dimensional scaling behavior is affected by logarithmic corrections. This suggests that $d=5$ is the upper critical dimension of the depinning transition in the random-field Ising model. Furthermore, we investigate the so-called creep regime (small driving fields and temperatures) where the interface velocity is given by an Arrhenius law.
\end{abstract}

Keywords: Disordered media, Driven interfaces, Numerical investigations, Non-equilibrium phase transitions, Creep motion

\section{INTRODUCTION}

Using the random-field Ising model (RFIM) we have studied the dynamics of driven interfaces in disordered ferromagnetic systems by means of Monte Carlo simulations. The interface separates regions of opposite magnetization and it is driven by a homogeneous field $H$. Without thermal fluctuations $(T=0)$ its dynamics is affected by the disorder in the way that the interface moves only if a sufficiently large driving field is applied. With decreasing driving field the interface velocity vanishes at a critical threshold $H_{\mathrm{c}}$ and is pinned below $H_{\mathrm{c}}$, due to the random-field. The field dependence of the interface velocity is given by

$$
v(h) \sim h^{\beta}
$$

with $h=\left(H-H_{\mathrm{c}}\right) / H_{\mathrm{c}} \geq 0$, i.e. this pinning/depinning transition can be considered as a continuous phase transition. A sketch of this behavior is shown in Fig. 1.

*Email: lars@thp.uni-duisburg.de 


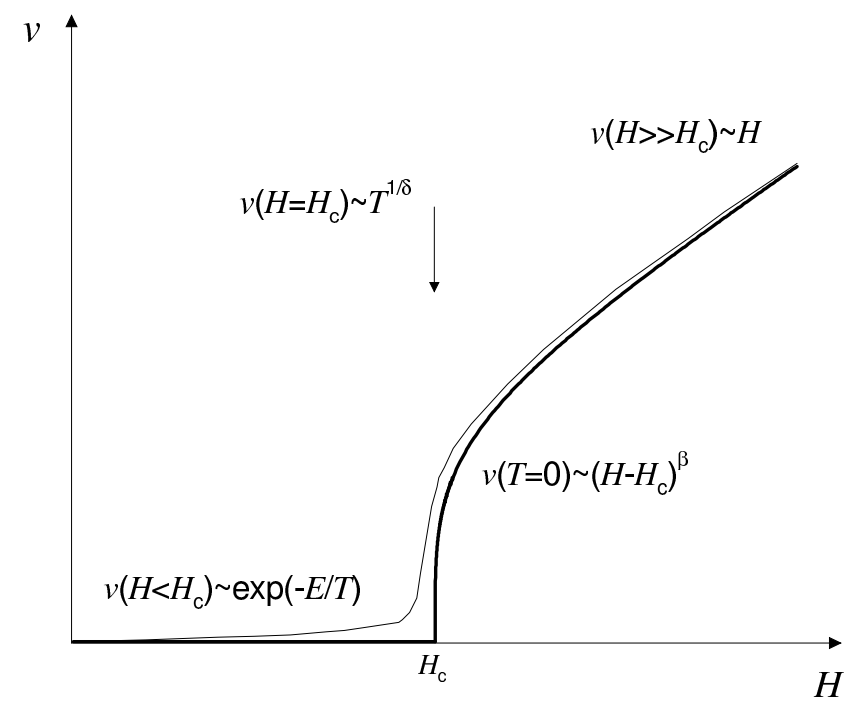

Figure 1: Sketch of the interface velocity $v$ on the driving field $H$ and temperature $T$. The bold line corresponds to $T=0$. With increasing temperature the depinning transition is smeared out (thin solid line). In the creep regime the interface velocity obeys an Arrhenius law.

The paradigm of the depinning transition is the Edwards-Wilkinson equation (Edwards and Wilkinson, 1982) with quenched disorder (QEW). The critical exponent $\beta$ of the QEW equation has been estimated within an renormalization group approach Nattermann, Stepanow, Tang and Leschhorn, 1992) It is expected that the critical behavior of both the RFIM and the QEW equation is characterized by the same exponents, i.e. both models are in the same universality class see e.g. (Bruinsma and Aeppli, 1984; Amaral, Barabási and Stanley, 1994).

In this paper we determine numerically the critical exponent $\beta$ of the depinning transition in the RFIM in various dimensions. We compare our results with those of the renormalization group approach mentioned above. In particular, we consider the five dimensional case where logarithmic corrections to the above scaling ansatz, Eq. (1), occur. These logarithmic corrections are a typical feature of the upper critical dimension where the so-called mean field solution becomes valid.

For finite temperatures no pinning of the interface occurs due to the thermal fluctuations. Even for driving fields well below $H_{\mathrm{c}}$ the interface is expected to exhibit a so-called creep motion, see Fig. 1, in which the interface velocity obeys an Arrhenius law. This Arrhenius law has been found for the QEW equation (Chauvé, Giamarchi and Doussal, 1998) and we compare these results with those of the RFIM. 


\section{THE RFIM MODEL}

In order to study the dynamics of moving interfaces numerically, we have investigated the $d$-dimensional RFIM which is characterized by the Hamiltonian,

$$
\mathcal{H}=-\frac{J}{2} \sum_{\langle i, j\rangle} S_{i} S_{j}-H \sum_{i} S_{i}-\sum_{i} h_{i} S_{i} .
$$

The sum in the first term is taken over all pairs of neighbored spins. It describes the exchange interaction between neighboring spins $\left(S_{i}= \pm 1\right)$ and a parallel alignment of nearest neighbors is energetically favored. Additionally, the spins are coupled to a homogeneous driving field $H$ and to a quenched local random-field $h_{i}$ with $\left\langle h_{i} h_{j}\right\rangle \propto \delta_{i j}$ and $\left\langle h_{i}\right\rangle=0$. The probability density $p$ that the local random-field takes some value $h_{i}$ is given by

$$
p\left(h_{i}\right)=\left\{\begin{array}{ccl}
(2 \Delta)^{-1} & \text { for } & \left|h_{i}\right|<\Delta \\
0 & & \text { otherwise }
\end{array}\right.
$$

i.e. $h_{i}$ is uniformly distributed. Throughout the paper we consider the case $\Delta=1.7$. Using both simple cubic (sc) and body centered cubic (bcc) lattices with antiperiodic boundary conditions we started each simulation for an initially flat interface. In non-disordered systems $(\Delta=0)$ the interface moves for any finite driving field. This limiting behavior for $\Delta \rightarrow 0$ can be recovered if the interface moves along the diagonal direction of simple cubic (sc) lattices or along the $\hat{z}$-direction of body centered cubic (bcc) lattices, for details see (Nowak and Usadel, 1998).

In our Monte Carlo simulations the dynamics of the interface motion is given by a Glauber dynamics with transition probabilities according to a heat-bath algorithm, see e.g. (Binder and Heermann, 1997). The interface velocity is the basic quantity in our investigations. Since a moving interface corresponds to a magnetization $M$ which increases with time $t$ (given in Monte Carlo steps per spin) the interface velocity is obtained according to $v \sim\langle\mathrm{d} M / \mathrm{d} t\rangle$, where $\langle\ldots\rangle$ denotes an appropriate disorder average.

\section{CRITICAL INTERFACE DYNAMICS}

In the following we analyse the interface dynamics in the vicinity of the critical threshold $H_{\mathrm{c}}$ at zero temperature $(T=0)$ on a bcc lattice. The interface velocity as a function of the driving field for the three dimensional model is shown in Fig. 2. The data suggest that $v$ vanishes continuously at the critical threshold $H_{\mathrm{c}}$. Fitting the data to the above ansatz, Eq. (1), one obtains $\beta=0.642 \pm 0.06$ and $H_{\mathrm{c}}=1.357 \pm 0.008$. This agrees with previous results. In a simple cubic lattice an exponent $\beta=0.6 \pm 0.11$ was observed for an interface moving along the [001] direction (Amaral, Barabás] and Stanley, 1994), and $\beta=0.66 \pm 0.04$ was observed for an interface moving along the [111] direction (Roters et al., 1999), respectively. Additionally, 


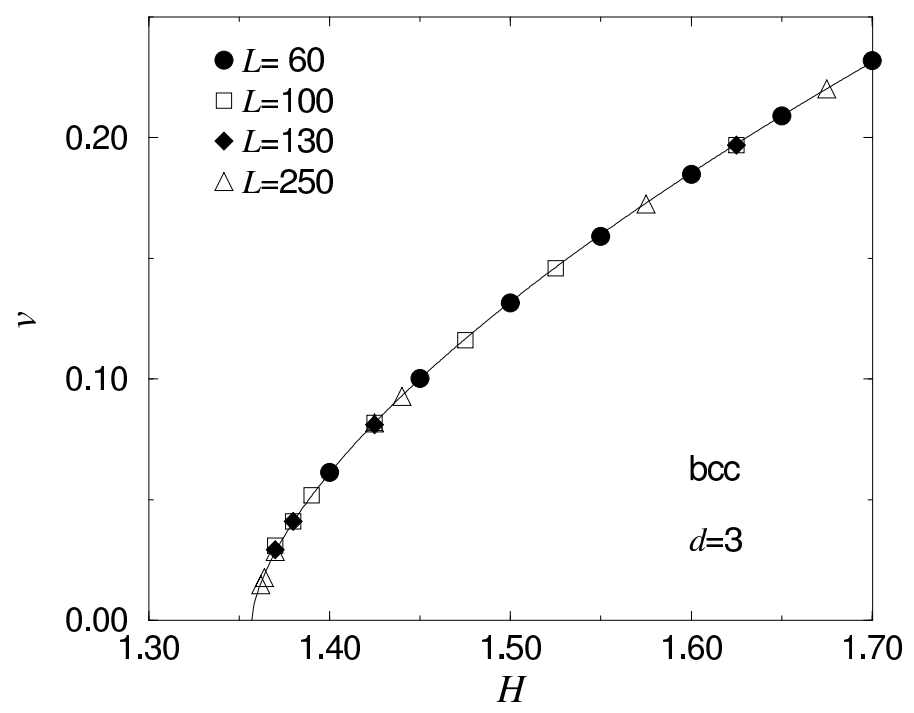

Figure 2: Dependence of the interface velocity $v$ on the driving field $H$ in the three dimensional model for different system sizes $L$. The data can be fitted (dashed line) according to Eq. (1). From the fit one obtains $H_{\mathrm{c}}=$ $1.357 \pm 0.008$ and $\beta=0.642 \pm 0.06$.

these values agree within the error-bars with the value $\beta=2 / 3$ found for the QEW equation with quenched disorder Nattermann, Stepanow, Tang and Leschhorn, 1992).

The above scaling behavior of the interface velocity, Eq. (11), is also found in two (Nowak and Usadel, 1998) and four dimensions (not shown). But in five dimensions the simple scaling ansatz Eq. (1) does not describe the velocity-field dependence in a convincing manner. In the inset of Fig. 3 we show the logarithmic derivative $\beta_{\mathrm{eff}}=\partial \ln v / \partial \ln h$ for various driving fields. Instead of a saturation for $h \rightarrow 0$ the effective exponent $\beta_{\text {eff }}$ displays a strong curvature which contradicts the scaling ansatz in Eq. (四).

In the following we will show that the data for $d=5$ are consistent with the assumption that the scaling behavior is given by Eq. (1) modified by logarithmic corrections. Usually logarithmic corrections affect the scaling behavior at the upper critical dimension $d_{\mathrm{c}}$. Below $d_{\mathrm{c}}$ the critical exponents depend on the dimension of the system while for $d>d_{\mathrm{c}}$ they are independent of the particular dimension and are given by those of the mean field theory. At $d_{\mathrm{c}}$ itself the mean field scaling behavior is modified by logarithmic corrections. The above mentioned renormalization group approach to the QEW equation predicts the values $d_{\mathrm{c}}=5$ and $\beta_{\mathrm{MF}}=1$.

Inspired by the usual scaling behavior at the critical dimension obtained from renormalization group approaches, see e.g. (Brézin, Le Guillou and Zinn-Justin, 1976), we assume that the asymptotic $(h \rightarrow 0)$ scaling behavior of the velocity is given in leading order by

$$
v \sim|h|^{\beta_{\mathrm{MF}}}|\ln h|^{x_{h}} .
$$




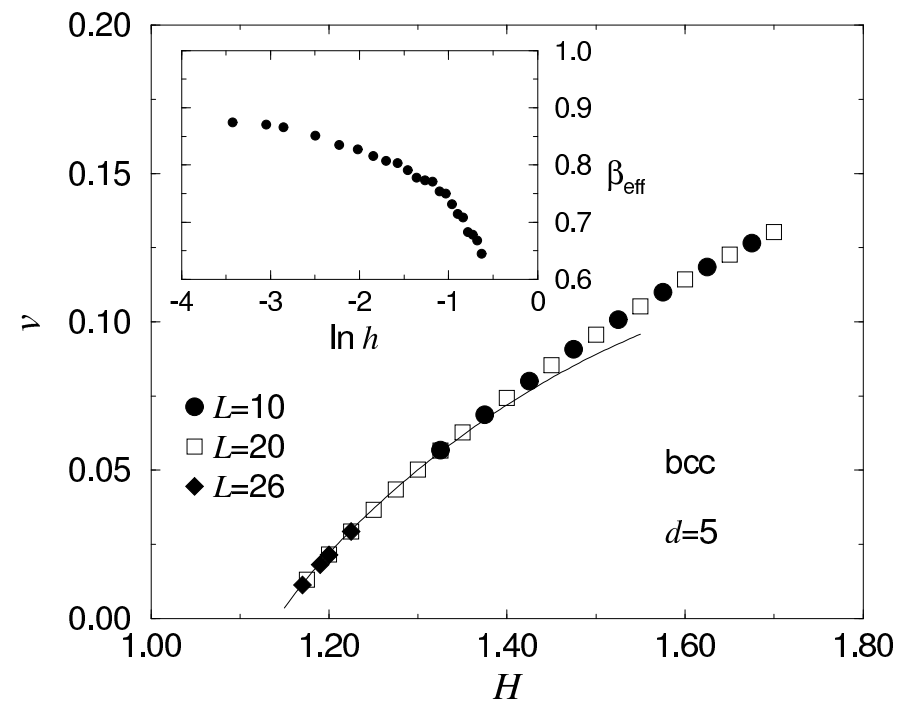

Figure 3: Dependence of the interface velocity $v$ on the driving field $H$ in the five dimensional model for different system sizes $L$. The dashed line is a fit according to Eq. (4) where $\beta_{\mathrm{MF}}=1$ has been used. As can be seen from the data, the fit works quite well for $H \rightarrow H_{\mathrm{c}}$ and one obtains $H_{\mathrm{c}}=$ $1.1425 \pm 0.001$ and $x_{h}=0.42 \pm 0.1$. The inset shows the logarithmic derivative $\beta_{\text {eff }}=\partial \ln v / \partial \ln h$. The strong curvature indicates that the velocity field dependence cannot be described by Eq. (11).

with $\beta_{\mathrm{MF}}=1$ and some unknown exponent $x_{h}$. The corresponding data as well as the fit according to Eq. (44) are shown in Fig. 3. From the fit which works for small values of $h$ we obtain $H_{\mathrm{c}}=1.143 \pm 0.01$ and $x_{h}=0.42 \pm 0.1$. It remains to be shown that the scaling behavior above $d_{c}$ is given by the pure mean field scaling behavior. Simulations of the six dimensional model are in progress and the results will be published elsewhere.

\section{THE CREEP MOTION}

In the previous section we focused our attention to the critical behavior at zero temperature. Including thermal fluctuations no pinning of the interface takes place, see Fig. 1. For example, at $H=H_{\mathrm{c}}$ the velocity grows with the temperature according to $v \sim T^{1 / \delta}$ (Nowak and Usadel, 1998; Roters et al., 1999). The interface moves even for driving fields well below the critical threshold. In this case the interface velocity is expected to obey an Arrhenius law

$$
v \sim C(H, T) \exp \left[-\frac{E(H)}{T}\right]
$$

which is characterized by its prefactor $C(H, T)$ and an effective energy barrier $E(H)$. Recently (Chauvé, Giamarchi and Doussal, 1998) investigated 


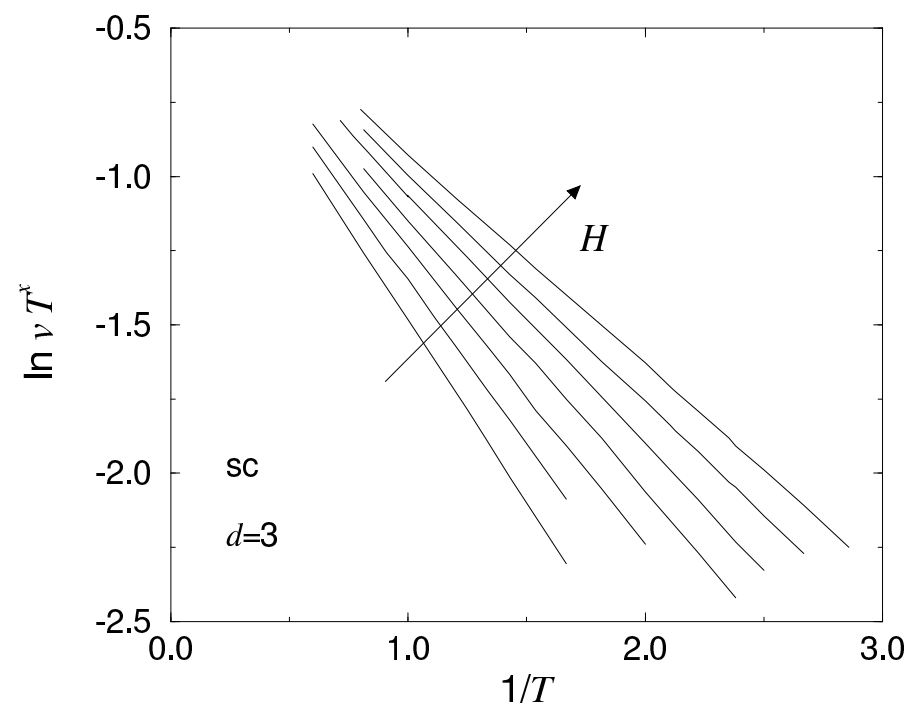

Figure 4: Interface velocities obtained at different driving fields $H=$ $0.3,0.35,0.4, \ldots, 0.6$ and rescaled according to Eq. (5) and (6). Varying $x$ we obtain nearly straight lines for $x=0.79 \pm 0.09$.

the creep regime in the QEW equation and found that the prefactor of the Arrhenius law is given by

$$
C(H, T)=c(H) T^{-x}
$$

with $c(H)$ being some function of the driving field. The temperature dependence is characterized by an exponent $x$. We apply this ansatz to our numerically obtained interface velocities in the $3 d$ RFIM. Figure 1 shows the data rescaled according to Eq. (5) and (6). As can be seen from the data, we obtain nearly straight lines for $x=0.79 \pm 0.09$ suggesting that the temperature dependence of the prefactor in Eq. (5) as well as the Arrhenius law itself are a proper description of the velocity in the creep regime. The slope of the lines in Fig. T corresponds to the effective energy barrier $E(H)$. Plotting $\ln v t^{x}$ vs. $E(H) / T$ the data collapse onto a single curve, see Fig. 5, without making any assumption about $c(H)$. This suggests that $c(H) \approx$ const holds.

However, details of our results do not coincide with those of Chauvé, Giamarchi and Doussal, 1998). In particular, these authors observed a significant field dependence of the prefactor $c(H) \sim H^{\mu}$ with $\mu>0$ and found a negative exponent $x$. Especially the opposite sign of $x$ is remarkable.

\section{SUMMARY}

We investigated the dynamics of driven interfaces for the random-field Ising model (RFIM). In our analyses we focus on the field and temperature dependence of the interface velocity and we consider the depinning transition 


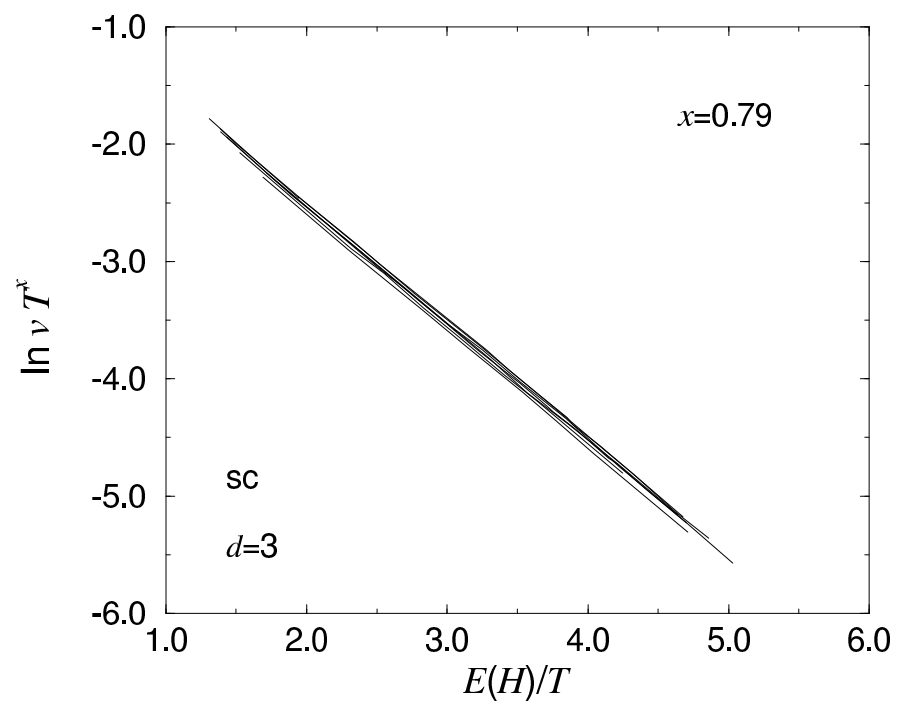

Figure 5: Rescaled interface velocities. The data coincide with those shown in Fig. 1 . They are rescaled with the numerically determined energy barrier $E(H)$. The coalescence of the data suggests $c(H) \approx$ const.

especially at temperature $T=0$. In the three dimensional model we find that $v(H)$ obeys a power law characterized by an exponent $\beta$. In $d=5$ the scaling behavior can be described by the mean field exponent modified by logarithmic corrections. This suggests that the upper critical dimension of the depinning transition in the RFIM is $d_{\mathrm{c}}=5$.

We also investigate the interface dynamics in the so-called creep regime which occurs for driving fields well below the critical threshold and small but finite temperatures. As predicted by a renormalization group approach the creep behavior is characterized by an Arrhenius law. But the details of the Arrhenius law differ from the predicted behavior.

\section{Acknowledgements}

This work was supported by the DFG via GK 277 Struktur und Dynamik heterogener Systeme (University of Duisburg) and SFB 491 magnetische Heteroschichten: Struktur und elektronischer Transport (Universities of Duisburg and Bochum).

\section{References}

Amaral, L. A., A.-L. Barabási and H. E. Stanley (1994). Universality class for interface growth with quenched disorder. Phys. Rev. Lett., 73, 62.

Binder, K. and D. W. Heermann (1997). Monte Carlo Simulation in Statisti- 
cal Physics, volume 80 of Springer Series in Solid-State Sciences. Springer, Berlin.

Brézin, E., J. C. Le Guillou and J. Zinn-Justin (1976). Field theoretical approach to critical phenomena. In C. Domb and M. S. Green, editors, Phase Transitions and Critical phenomena, volume 6, 125. Academic Press, London.

Bruinsma, R. and G. Aeppli (1984). Interface motion and nonequilibrium properties of the random-field Ising model. Phys. Rev. Lett., 52, 1547.

Chauvé, P., T. Giamarchi and P. L. Doussal (1998). Creep via dynamical functional renormalization group. Europhys. Lett., 44, 110.

Edwards, S. F. and D. R. Wilkinson (1982). The surface statistics of a granular aggregate. Proc. R. Soc. London A, 381, 17.

Nattermann, T., S. Stepanow, L.-H. Tang and H. Leschhorn (1992). Dynamics of interface depinning in a disordered medium. J. Phys. II, 2, 1483.

Nowak, U. and K. D. Usadel (1998). Influence of the temperature on the depinning transition of driven interfaces. Europhys. Lett., 44, 634.

Roters, L., A. Hucht, S. Lübeck, U. Nowak and K. D. Usadel (1999). Depinning transition and thermal fluctuations in the random-field Ising model. Phys. Rev. E, 60, 5202. 